\section{La Révolution française}

Cahiers de l'Institut d'histoire de la Révolution française

$20 \mid 2021$

La Révolution en 3D - Textes, images, sons

(1787-2440)

Olympe de Gouges et la scène, ou le « devenir théâtre » d'olympe de Gouges (imaginaires théâtraux XIX-XXI)

\title{
Sophie Lucet
}

\section{OpenEdition}

\section{Journals}

Édition électronique

URL : https://journals.openedition.org//rf/4924

DOI : $10.4000 /$ Irf.4924

ISSN : 2105-2557

Éditeur

IHMC - Institut d'histoire moderne et contemporaine (UMR 8066)

Référence électronique

Sophie Lucet, « Olympe de Gouges et la scène, ou le « devenir théâtre » d'Olympe

de Gouges (imaginaires théâtraux xIx-xxI) », La Révolution française [En ligne], 20 | 2021, mis en ligne le 25 janvier 2021, consulté le 29 juin 2021. URL : http://journals.openedition.org/lrf/4924 ; DOI : https:// doi.org/10.4000/Irf.4924

Ce document a été généré automatiquement le 29 juin 2021.

(c) La Révolution française 


\title{
Olympe de Gouges et la scène, ou le " devenir théâtre " d'Olympe de Gouges (imaginaires théâtraux XIX-XXI)
}

\author{
Sophie Lucet
}

\section{NOTE DE L'ÉDITEUR}

Cet article est basé sur une communication à trois voix présentée lors du colloque « La Révolution en 3D - Textes, images, sons (1787-2440) » qui s'est tenu à l'université Paris 1 Panthéon-Sorbonne du 14 au 16 mars 2019, organisé par le Cespra et l'IHMC-IHRF. Vous pouvez retrouver cette communication sur la chaîne YouTube de l'IHMC à l'adresse : https://youtu.be/21p_I6mYDn8

" Je suis pétrie de petits défauts; mais je possède de grandes vertus. Peu de personnes me connaissent à fond, peu sont en état de m'apprécier ; on a eu différentes disputes sur mon compte. Les uns me voient d'une façon, chacun me juge différemment et je suis cependant toujours la même. Ce n'est pas moi qui varie ; je ne puis sympathiser qu'avec des personnes véritablement honnêtes [...] Et quand je parviendrais à une célébrité que je ne puis espérer, on me verra toujours cette même simplicité que j'ai eue avant d'être Auteur ». 


\section{Introduction}

1 Qu'on le définisse comme genre littéraire ou comme lieu de sociabilité, comme espace de représentation ou comme instance de légitimation littéraire, comme tréteau de parade d'un soi hypostasié ou comme tribune d'idées, le Théâtre dans toutes ses potentialités semble bien constituer l'un des prismes dans lequel peut se penser, s'apercevoir, s'imaginer la figure labile d'une Olympe de Gouges, décriée autant que célébrée dans le cours du long processus de son intégration à notre histoire.

2 Sa vocation tôt déclarée pour le théâtre fut-elle le moyen pour Olympe de Gouges de réaffirmer envers et contre tout son lien de filiation avec le dramaturge Lefranc de Pompignan ? Ou bien fut-ce plutôt l'effet et le résultat d'une « théâtromanie » d'époque à laquelle la vie galante et mondaine d'olympe de Gouges à Paris l'avait «naturellement » exposée? Tout cela ensemble, très probablement, entrait dans ce «tropisme » théâtral d'une Olympe de Gouges qui fréquenta assidûment les salles de spectacle parisiennes avant la Révolution, participa en tant qu'actrice à des représentations de théâtre de société, se lança enfin dans la rédaction d'œuvres dramatiques, où elle se plut à mettre en scène non seulement ses idées et ses combats, mais aussi parfois son personnage, de manière implicite ou explicite ${ }^{2}$, faisant du théâtre un espace où déployer fictivement la dynamique d'une conquête et d'une émancipation, tout comme l'invention d'une parole politique : il fallait bien cela sans doute pour préparer une entrée en scène autrement problématique sur le théâtre de l'Histoire, où on ne l'attendait pas davantage, mais où elle s'engagea avec audace - très courageusement pour les uns, impudemment pour les autres - jusqu'à perdre sa vie sur la Place de la Révolution le 3 novembre 1793.

3 "Théâtrale ", Olympe de Gouges l'était sans doute à plus d'un titre, et cette proximité avec la scène et la parole adressée permet peut-être d'appréhender sous un autre angle la singularité de ses moyens d'expression politique: préfaces, lettres, brochures, placards, campagnes d'affiches ${ }^{3}$. De nombreux témoignages s'accordent du reste sur le franc-parler du personnage et son usage de la parole qui n'était pas celui d'une oratrice impeccable (elle n'avait pas reçu toute la formation rhétorique nécessaire), mais celui d'une intarissable discoureuse, habile aussi aux mots d'esprit. Un autre aspect de cet éthos théâtral du personnage se révèle ainsi dans la foncière oralité de ses textes, et dans son rapport second à la langue française et à l'écrit; l'occitan était sa langue maternelle ${ }^{4}$ : Olympe de Gouges, comme elle le rappelle elle-même dans plusieurs textes, eut recours à de nombreux «secrétaires $»^{5}$; elle se prévaut également d'une grande rapidité de composition que lui permettait cette manière de faire sténographier ses textes; et il semblerait de fait qu'une part importante de sa production écrite ait été dictée à des copistes ${ }^{6}$,

4 Cette théâtralité qui traverse l'œuvre et la vie d'olympe de Gouges - au-delà de ses activités littéraires et dramatiques - a contribué sans conteste à nourrir diversement la légende posthume du personnage, que l'on se réfère à la mauvaise réputation que lui a faite un XIX $x^{e}$ siècle, prompt aussi à l'oublier, ou que l'on se tourne vers les portraits idéalisés que retient parfois la vulgate féministe des $\mathrm{xx}^{\mathrm{e}}$ et $\mathrm{xxI}^{\mathrm{e}}$ siècles dans le processus d'une iconisation qui peut devenir « neutralisante».

5 Sans nulle intention de nous mesurer ici à ses biographes et à ses meilleurees historien'n'e's, c'est à la question des représentations imaginaires de cette Olympe de Gouges théâtrale que nous nous sommes intéressée dans l'étude qui suit ${ }^{7}$, en 
proposant tout d'abord, dans nos deux premiers développements, un diptyque contradictoire rapidement brossé : d'une part, nous revenons sur certains éléments de la «légende noire » d'olympe de Gouges, et sur le processus d'une regrettable oblitération, au XIX ${ }^{e}$ siècle, de son œuvre (théâtrale notamment) et de son action; d'autre part, sur le versant des réhabilitations contemporaines, nous examinons brièvement trois spectacles récents, illustrant un «devenir théâtre " d'Olympe de Gouges, dans les formes parfois convenues de l'héroïsation et de la célébration d'une pionnière de l'histoire des femmes. Dans le troisième temps de ce travail - le plus conséquent parce qu'il s'attache à présenter une archive originale -, nous assumons le choix d'une présentation non chronologique de notre propos, en revenant vers une Olympe théâtrale du XIX $x^{e}$ siècle - inaperçue jusqu'alors et découverte dans le cours de notre enquête - dont la figure, très librement réinventée, traverse un spectacle fin-desiècle oublié et qui mérite pourtant, à plus d'un titre, d'être replacé dans la longue séquence des représentations de la Révolution française sur les scènes parisiennes au $\mathrm{XIX}$ siècle : Camille Desmoulins, drame en cinq actes et huit tableaux d'Émile Moreau, créé le $1^{\text {er }}$ avril 1879 au Théâtre-Historique (rebaptisé alors Théâtre des Nations). Dans ce drame historique sur la Révolution - et d'obédience républicaine -, inspiré à la fois par l'histoire documentaire et la fantaisie romanesque, l'auteur fait surgir une Olympe de Gouges fantasque, impertinente et prophétique, d'une efficacité théâtrale que ne lui pardonnent pas les critiques contemporains, inquiets du retour inattendu de cette figure refoulée par l'Histoire et qui vient avant l'heure réclamer ses droits dans une fiction de théâtre.

\section{La mauvaise réputation : aperçu des aléas de la mémoire théâtrale d'Olympe de Gouges au XIX ${ }^{\mathrm{e}}$ siècle.}

6 La personnalité bouillonnante d'olympe de Gouges, sa trajectoire de vie mouvementée et sa fin tragique, ses ambitions et ses diverses expériences théâtrales, son œuvre dramatique publiée enfin, tout cela semblait devoir assurer à Olympe de Gouges une forme de visibilité, sinon de reconnaissance, dans l'histoire du théâtre. Cela faisait d'elle à tout le moins un masque " comique ${ }^{8}$ » historiquement identifié, notamment et tout d'abord pour ses contributions attestant la vitalité de la vie théâtrale avant et pendant la Révolution, à travers ses différentes pièces composées entre 1784 et 1793. Comédies d'intrigues et comédies de mœurs, pièces d'actualités et pièces de circonstances, elles rencontrèrent un accueil contrasté au cours de cette brève décennie, au fil de leur publication et selon leur acceptation ou non par le comité de lecture de la Comédie-Française et d'autres théâtres, au gré aussi du succès ou de l'échec de leurs représentations.

$7 \quad$ Le cadre limité et la perspective du présent article ne nous permettent pas de nous arrêter sur la genèse et l'étude détaillé de ce corpus varié et passionnant, - lequel témoigne de la remarquable productivité d'olympe de Gouges dans le domaine du théâtre, de sa conviction qu'il y avait là une tribune de choix pour exprimer des idées neuves, enfin de la ténacité dont elle fit preuve pour parvenir à imposer certaines de ses œuvres à la scène, en revendiquant une auctorialité pleine et entière dans le domaine du théâtre, elle qui était autodidacte, elle qui était femme; elle le rappelle d'ailleurs à plusieurs reprises dans les préfaces de ses œuvres littéraires. Dans cet ensemble, plus vaste qu'il n'y parait d'abord - entre les textes publiés et les inédits, les 
œuvres en cinq actes, trois actes, un acte, les manuscrits perdus et les projets non réalisés - se recommandent à l'attention notamment les pièces où la dramaturge se mesure aux grands auteurs et aux contemporains célèbres (Le Mariage inattendu de Chérubin; Le Nouveau Tartuffe ou l'école des jeunes-gens; Molière chez Ninon ou le siècle des grands hommes), les pièces de genre - comédies et drames (L'homme généreux; Lucinde et Cardénio, ou Le Fou par amour; Le Philosophe corrigé, ou le cocu supposé...) -, celles où elle s'attaque aux préjugés et aux injustices sociales (Zamore et Mirza ou l'heureux naufrage première version de L'esclavage des Nègres; La Nécessité du divorce; Le Couvent ou les vœux forcés...), les pièces d'actualité politique enfin (Mirabeau aux Champs-Elysées; Les Aristocrates, ou les curieux du Champ de Mars ; La France sauvée ou le Tyran détrôné...). Dans leur diversité, les ambitions affichées, et les circonstances diverses de leur réception, avant et pendant la Révolution, ces pièces - qui furent en leur temps critiquées, soutenues, empêchées ou moquées - semblent bien mériter pourtant d'être considérées comme les éléments d'une œuvre singulière et identifiable, historiquement inscrite dans le contexte du théâtre de la fin du XVIII ${ }^{e}$ siècle, ainsi que le démontrent aujourd'hui plusieurs études décisives et les éditions scientifiques des textes dramatiques d'Olympe de Gouges".

Olympe de Gouges eut certes des difficultés à faire jouer ses pièces - un bon nombre d'entre elles ne passèrent pas le cap des comités de lecture (celui de la ComédieFrançaise notamment) -, elle dut également batailler (et payer de ses deniers) pour publier ses œuvres ${ }^{10}$, certaines demeurant finalement à l'état de manuscrit ou de projet. Mais plusieurs de ces pièces forcèrent l'attention des contemporains par leur intérêt et l'audace de leur propos, à commencer par la plus célèbre d'entre elle - dénonçant le scandale humain de l'esclavage -, L'Esclavage des noirs, ou l'heureux naufrage, dont une première version fut acceptée dès 1785, puis bloquée par les Comédiens Français, avant d'être jouée finalement le 27 décembre 1789, non sans polémique et cabale, du fait de son sujet et de la résistance d'une partie du public liée aux propriétaires des colonies. L'on doit retenir aussi le succès de sa pièce portant sur l'enfermement conventuel des femmes, Le Couvent, ou les vœux forcés, qui fut également créée au Théâtre Français, le 21 octobre 1790, et connut plus de quatre-vingt représentations si l'on en croit l'édition de $1792^{11}$. On peut également citer L'Entrée de Dumouriez à Bruxelles, ou les Vivandiers, représentée le 23 janvier 1793 au Théâtre de La Nation, sans doute un échec, mais qui témoigne de l'activisme politique d'Olympe de Gouges dans ces années périlleuses. Or, dans ces cas particuliers, il semble que l'histoire ait surtout et durablement retenu le souvenir de "fours » cuisants, laissant, à tort, croire à l'échec des diverses tentatives théâtrales d'une "dramaturge d'occasion", présentée comme opportuniste et inopportune. De fait, pour la majeure partie de son œuvre théâtrale et littéraire, il apparait bien que la postérité d'olympe de Gouges ait durablement souffert d'une réputation fâcheuse de "mauvais auteur » transmise par ses détracteurs et ses rivaux, et des témoignages qui ont nourri une historiographie négative et vite oublieuse tout au long du XIX ${ }^{e}$ siècle et jusqu'au $\mathrm{Xx}^{\mathrm{e}}$ siècle. La chronique et l'histoire des théâtres ont retenu principalement les témoignages à charge d'une critique mal disposée, ou bien encore les calomnies de ses ennemis politiques de tous bords. Ainsi, dans nombre d'études portant sur le théâtre à l'époque de la Révolution, ses pièces sont bien souvent expédiées en quelques lignes, les auteurs s'appuyant les uns après les autres sur les mêmes relations partialement négatives et sur les mêmes appréciations misogynes, empruntées notamment à ses meilleurs ennemis, les Comédiens Français: dans L'Histoire du Théâtre Français pendant la Révolution d'Étienne et Martinville ${ }^{12}$, entres 
autres mentions dénigrantes, elle surgit en « femme, vieille et laide ${ }^{13}$ » venant protester contre le mauvais zèle des comédiens; de même dans les Mémoires (apocryphes) de Fleury ${ }^{14}$ où, plus longuement, et au fil d'un exercice satirique parfois savoureux, elle est portraiturée en « cresselle du Parnasse » et en « méchant auteur » ${ }^{15}$.

9 Sur la question d'une réception du théâtre d'Olympe de Gouges par ses contemporains et celle de sa postérité au XIX siècle, l'on s'en tiendra ici à un bref échantillon, emblématique du discrédit constamment attaché à la figure d'olympe de Gouges, bien au-delà de son théâtre, et touchant aussi, en réalité, à la légitimité même de son combat politique. Ainsi, lors de la création, en 1793, de la grande pièce républicaine consacrée par l'autrice à L'Entrée de Dumouriez à Bruxelles, Guénégaud dénonce-t-il, dans Le Journal Français du 25 janvier, « une rhapsodie à sa façon » et « un monstre dramatique ». Les Petites Affiches ne jugent pas mieux « cet ouvrage bizarre, dans lequel on ne trouve ni plan, ni conduite, ni goût, ni rien de ce qui constitue la véritable comédie ». Et, dans sa recension de La Correspondance littéraire, Grimm ne ménage pas davantage " un ouvrage aussi étrangement mauvais ", "monstrueux ", " où l'ignorance des premières règles dramatiques ne le cédait qu'au mauvais goût du dialogue et à l'obscénité des expressions qui souvent ont fait rougir même la pudeur révolutionnaire ». À la suite de ces premiers censeurs, tout au long du XIX ${ }^{e}$ siècle et jusque dans les années 1960, les mêmes récits désobligeants puisés aux mêmes sources et repris, parfois au mot près (et sans guillemets), nourrissent la plupart des études consacrées au théâtre de la période révolutionnaire, depuis l'ouvrage d'Étienne et Martainville ${ }^{16}$, jusqu'au Théâtre de la Révolution française, de Marvin Carlson ${ }^{17}$ (1966 et 1970), en passant par Le Théâtre Révolutionnaire d'Eugène Jauffret, publié en $1869^{18}$.

Même lorsque, après 1848, elle commence à être présentée sous un jour plus favorable à défaut d'être véritablement prise au sérieux -, notablement dans l'ouvrage de l'historien Capefigue, Les Déesses de la Liberté. Les femmes de la Convention et du Directoire, publié en $1862^{19}$, et de manière plus décisive (et moins réactionnaire) dans celui de Charles Monselet, Les Oubliés et les dédaignés du dix-huitième siècle, publié en $1876^{20}$-, Olympe de Gouges n'échappe pas toujours aux discours de la misogynie ordinaire des hommes du XIX siècle.

11 Monselet, parmi ses "oubliés» du XVIII siècle, lui consacre ainsi une quinzaine de pages de pages bien documentées, interrogeant les raisons de sa disparition : «Sa vie, une des plus haletantes et des plus dramatiques, étonne, et fait qu'on se demande comment tant de silence a remplacé tant de bruit ${ }^{21}$. » Au fil de ce récit, que l'on peut considérer comme l'une des premières biographies «dramatiques" fiables d'olympe de Gouges, donnant substance à la femme de théâtre et à l'activiste politique, Monselet ne peut s'empêcher pourtant de dénigrer sa vocation, parlant du " démon des lettres » qui la pousse à devenir "la Sappho de son siècle ", de ses "foucades littéraires ", du genre du «drame » enfin « vers lequel sa nature la poussait irrésistiblement $»^{22}:$ «Il ne fallut rien moins qu'une révolution pour mettre en lumière, elle et ses drames » rajoute l'historien, qui revient constamment sur les faibles qualités littéraires de cette « amazone littéraire » dont il admire pourtant l'esprit et le courage : « On se surprend à souhaiter un peu plus de talent à cette malheureuse si cruellement mordue par la tarentule politique. $»^{23}$ Alphonse Daudet, à son tour, dans le compte-rendu substantiel qu'il consacre à l'ouvrage de Monselet, désigné comme « le très spirituel historien de la dame de Gouges ", met également en doute la vocation littéraire de celle qui « devient homme de lettres et écrivain dramatique, au moment où le plaisir et la galanterie allait 
renoncer à elle » : "Qu'était-ce donc que cette Olympe de Gouges joie et terreur des sociétaires du théâtre français? ", "d'où lui venait ce nom et ce panache », s'interroge encore l'écrivain, qui l'associe à « ces sortes de femmes brûlées par un éternel besoin de parade et de publicité » et en rajoute sur « la harceleuse » des Comédiens Français qui " durent subir la lecture successive de nombreux drames et mélodrames de l'infatigable "authoress" "24. Et, au cœur même de l'hommage pourtant rendu par l'écrivain à l'intrépidité politique du personnage et à son courage devant le Tribunal révolutionnaire, se reformule encore et toujours la condescendance virile de tout un siècle :

Olympe s'y tint avec dignité, s'entendit condamner sans pâlir, et livra courageusement à l'échafaud sa pauvre tête folle, ivre de bruit, de vanité, où le soleil de midi avait entretenu trop de combustions sourdes, d'ébullitions précipitées, pour qu'elle pût se plonger impunément dans l'atmosphère volcanique de la Révolution ${ }^{25}$.

12 Folle héroïque, folle littéraire, l'image construite d'olympe de Gouges - même lorsqu'elle se précise ${ }^{26}$ - porte ainsi, tout au long du xIx ${ }^{e}$ siècle et au-delà ${ }^{27}$, le stigmate d'un jugement relativisant l'intérêt de son œuvre (notamment théâtrale), comme la portée de ses idées sociales et politiques. Le fil souterrain d'une mémoire moins négative du personnage, allant de Michelet à Léopold Lacour, peut s'apercevoir néanmoins, comme nous nous proposons de le donner à voir - à partir d'une archive méconnue - dans la troisième partie du présent article. Mais il est frappant de relever, dans l'étude de Lacour-qui signe la première biographie sérieusement documentée d'Olympe de Gouges, publiée en 1900 et dédiée à Alphonse d'Aulard -, nombre de traits qui recueillent l'héritage de cette historiographie négative, alors même que son auteur prétend y dresser le portrait réhabilité d'une pionnière du féminisme ${ }^{28}$.

\section{De l'autrice au personnage : trois exemples théâtraux des célébrations d'Olympe de Gouges au XXI ${ }^{\mathrm{e}}$ siècle.}

Comme l'histoire de l'édition des textes d'olympe de Gouges, plus particulièrement ceux qu'elles destinaient à la scène, permet de le comprendre ${ }^{29}$ - par-delà la trajectoire de réhabilitation de cette figure de pionnière tout au long $d u x^{e}$ siècle $^{30}-$, il faut attendre le moment de reconnaissance des études féministes, depuis les années 1960 et jusqu'à aujourd'hui, pour voir apparaître dans le paysage éditorial des éditions critiques de son théâtre et, grâce à ce travail philologique, la possibilité d'une relecture de ces œuvres ${ }^{31}$. Quant à la postérité proprement scénique des pièces, elle parait quasi inexistante et problématique à plus d'un titre: hormis peut-être, et en de rares occasions, la pièce L'Esclavage des noirs, ou l'heureux naufrage, citée ou performée à l'occasion de commémorations concernant l'abolition de l'esclavage ${ }^{32}$, les autres pièces d'Olympe de Gouges, comme tant de répertoires oubliés, semblent n'être plus - sinon pour les chercheurs - que des objets théâtralement morts, frappés d'une inéluctable péremption, pour des raisons autant esthétiques qu'idéologiques ${ }^{33}$.

14 Le mouvement de reconnaissance de la figure d'Olympe de Gouges et de l'importance de ses combats politiques, passe - comme le montrent bien les études récentes qui lui sont consacrées - par l'histoire du féminisme au $\mathrm{xx}^{\mathrm{e}}$ siècle, et par un processus récent et remarquable d'iconisation de la rédactrice de la Déclaration des droits de la femme et de la citoyenne. 
15 Au théâtre, et dans cette perspective, l'on peut repérer, depuis le bicentenaire de la Révolution, mais surtout depuis les années 2000, un certain nombre de spectacles consacrés à la figure pionnière d'olympe de Gouges, envisagée sous l'angle d'une biographie tragique et d'un destin exemplaire, témoignant ainsi au nom de toutes les femmes en faveur d'une émancipation à conquérir. Le plus souvent, il s'agit de mises en scène signées par des femmes, parfois pour des "seules en scène ", mais surtout, et de manière récurrente, dans le geste d'une affirmation auctoriale féminine forte, jouant le plus souvent de l'identification et de la célébration.

16 Ces spectacles, non sans didactisme pour la plupart, témoignent également des renouveaux historiographiques et éditoriaux, indispensables à la reconsidération de la vie et des œuvres d'Olympe de Gouges. À des degrés divers, dans le travail de l'incarnation et de l'énonciation propre au théâtre, ils présentent certainement et surtout l'intérêt de tenter de faire valoir, au-delà de la seule illustration biographique, la singularité de la voix d'olympe de Gouges. Au bénéfice de leur relecture, ils mettent à l'épreuve du théâtre la force d'interpellation que recèlent nombre de ses textes et discours, issus d'une époque où il était si problématique pour les femmes de porter une parole politique légitime.

17 Dans l'ordre d'une chronologie récente, et à titre d'exemples, l'on peut tenter de décrire brièvement trois spectacles qui - entre 2008 et $2018^{34}$-illustrent, de manière emblématique à nos yeux, cette récurrence du personnage d'Olympe de Gouges sur les scènes contemporaines :

18 Terreur, Olympe de Gouges est l'adaptation pour le théâtre d'un texte de l'écrivaine et dramaturge Elsa Solal, qui en a consacré plusieurs à Olympe de Gouges depuis 2008 (notamment un album jeunesse intitulé Non à la discrimination des femmes chez Actessud $^{35}$ ), conçue et mise en scène par Sylvie Pascaud en octobre 2013 au Théâtre du Lucernaire. Le spectacle - repris avec succès à plusieurs reprises depuis 2015, et publié depuis ${ }^{36}$ - présente le jeu d'un trio, porté par les comédien-ne-s Anne-Sophie Robin, Martial Jacques et Gilles Nicolas. S'inscrivant plus directement que d'autres dans le genre de la pièce historique, l'action - située doublement la veille de l'arrestation d'Olympe de Gouges et au moment de son procès expédié et de sa condamnation à mort - présente l'intérêt d'une double mise en perspective, littéraire et politique, en plaçant la figure d'Olympe de Gouges entre celle de son confident, Louis-Sébastien Mercier, et celle de son persécuteur, Antoine Fouquier-Tinville. Le titre de la pièce et ce dispositif d'encadrement - le fait aussi que l'auteur choisisse de faire de Mercier l'amant d'olympe - dessinent le projet d'une interrogation parallèle portant sur les violences politiques de la Révolution, celles notamment faites aux femmes, et sur l'atavique guerre des sexes: où l'on voit Olympe récuser les conseils de modération de Mercier, puis affronter vaillamment et jusqu'à la mort un procès inique, en défendant éloquemment ses idées, et jusqu'à la rédaction de la dernière lettre qu'elle adresse à son fils et que le spectacle, soucieux de la précision de l'archive, fait entendre entièrement et littéralement ${ }^{37}$.

Olympe de Gouges, porteuse d'espoir, d'après les écrits d'olympe de Gouges est le titre d'un spectacle régulièrement repris jusqu'à ce jour, depuis sa création en septembre 2012, au Théâtre du Guichet Montparnasse, co-signé par la dramaturge Clarissa Palmer et par Annie Vergne, comédienne, metteuse en scène et directrice depuis 1986 de ce «théâtre de poche ", accueillant la pratique amateure comme la création. Le texte de la pièce, également co-écrit avec Clarissa Palmer - universitaire anglo-saxonne autrice d'une 
thèse sur Olympe de Gouges et traductrice de ses écrits en anglais - a fait l'objet d'une intéressante édition bilingue aux éditions de L'Harmattan ${ }^{38}$. Résolument conçue comme un hommage à la pionnière du féminisme et comme une réflexion soutenue par les gender studies, l'œuvre est interprétée par Annie Vergne, Juliette Stevez et Guislain Geiger : elle met en scène le personnage d'un jeune historien, thésard aux prises avec la biographie de Gouges, et pour qui vont se dédoubler, dans le temps d'une heure de spectacle, l'olympe du xVIII siècle et une Olympe imaginaire plus proche et plus complexe. Le projet ne manque pas d'ambition sur le terrain de l'historiographie, participant à la divulgation d'une œuvre mal connue, en évitant les pièges de la mythologisation naïve. Les quelques extraits accessibles sur l'internet ${ }^{39}$ prouvent, en outre, l'intérêt et l'originalité d'une approche théâtrale soucieuse d'interroger à la fois les postures de genres et le rôle de l'imagination dans le travail historique.

Enfin, dans une veine proche de la pièce précédente, mais plus intéressante par sa dramaturgie et par une écriture théâtrale mieux éprouvée, l'on doit s'arrêter ici sur l'œuvre récente de la comédienne, metteuse en scène et autrice Catherine Anne, et sur un titre qui retient aussi l'attention: J'ai rêvé la Révolution, "spectacle librement inspiré par la vie et la mort d'olympe de Gouges ", texte et mise en scène de Catherine Anne, co-mise en scène par Françoise Fouquet, créé en janvier 2018 à Grenoble (présenté en février 2018 à la Manufacture des (Eillets à Ivry, et en tournée depuis ${ }^{40}$ ). Il s'agit, cette fois, d'évoquer les derniers moments de la prisonnière à la suite de son procès, et avant sa mort, le lendemain, 3 novembre 1793. Le parti-pris dans le texte et dans la mise en scène consiste à montrer l'universalité d'une situation qui évoque la violence de l'Histoire, le courage des individus, la difficulté de la transmission. Ainsi, les personnages - un quatuor cette fois- ne sont-ils désignés que comme «la prisonnière ", « le jeune soldat », « la mère », et « la jeune femme épouse du fils de la prisonnière " ${ }^{41}$. L'enjeu consiste à faire entendre la voix et les idées d'Olympe de Gouges dans leur humanité, et à tenter de les rapprocher du spectateur dans une écriture "tendue entre l'Histoire et la violence de notre actualité ", comme l'écrit l'autrice, metteuse en scène, et comédienne dans sa note d'intention ${ }^{42}$. Gommées dans un premier temps, et dans le décor quelque peu «boltanskien » d'un mur de chemises blanches suspendues - qui sont comme celles de tous les morts des révoltes et des révolutions confisquées-, les références à la Révolution Française reviennent progressivement et en pointillé (une casquette remplacée par un bonnet phrygien, le scénario d'un complot de fuite esquissé et récusé, les cheveux coupés, le bruit du couperet). La voix d'Olympe de Gouges, jamais nommée, est une voix intérieure et intime, «la mémoire de sa voix, ou sa voix poétique », écrit encore Catherine Anne. La dramaturge a cherché manifestement à limiter les effets trop manifestes de citations et, partant, l'écueil de la déclamation éloquente et de la posture - au profit d'une évocation plus terriblement concrète et à nu de la réalité de l'histoire. De fait, ce n'est qu'à l'épilogue contemporain d'un dialogue entre deux femmes d'aujourd'hui, mère et fille, qu'il revient d'établir plus explicitement un lien avec l'histoire, dans son exemplarité, en faisant sa place à l'archive - celle de l'intime notamment, comme dans Terreur, Olympe de Gouges - en donnant à entendre la dernière lettre d'Olympe à son fils - le texte admirable d'un ferme et poignant adieu - et la voix de la mère qui jamais n'abdique au creux, ou au revers d'une parole publique marquée au sceau d'une sincérité en quelque sorte renchérie. Pas plus que dans le spectacle d'Elsa Solal, par ailleurs, Catherine Anne ne juge intéressant de revenir sur l'épisode, sinistre et anecdotique, du reniement d'Olympe par Pierre Aubry dans sa « Profession civique » du 
14 novembre 1793, préférant ainsi aux apories de l'approche biographique les termes d'un postlude théâtral et contemporain à usage des nouvelles générations ${ }^{43}$.

\section{Dans le drame historique du XIX ${ }^{\mathrm{e}}$ siècle : Olympe de Gouges, le personnage en trop.}

21 Pour achever cet aperçu des destinées d'Olympe de Gouge au théâtre - et boucler la boucle en quelque sorte en revenant aux scènes $\mathrm{du}_{\mathrm{XIX}} \mathrm{x}^{\mathrm{e}}$ siècle - il nous reste à présent à remonter dans le temps et à nous étonner qu'Olympe de Gouges n'ait apparemment suscité au fil du siècle aucun drame dont elle serait l'héroïne. Tel n'est pas le cas des Charlotte Corday, Madame Roland, ou Madame Tallien, dont on peut repérer les occurrences dans le répertoire et la titrologie du théâtre du XIX , parmi les nombreuses pièces qui traitent de la période révolutionnaire, notamment après 1848 et dans les débuts de la III $^{e}$ République ${ }^{44}$. Cette absence d'Olympe de Gouges sur les scènes de théâtre au XIX donne sans doute la mesure à la fois de l'excès et du scandale qu'elle représentait alors. Incontestablement, l'autrice de La Déclaration des droits de la femme et la défenseuse auto-désignée de Louis XVI sentait le souffre : trop républicaine pour les uns, trop royaliste pour les autres, incohérente ou folle pour certains, "mauvais auteur " pour la plupart, virago et harceleuse de comédiens, elle dérangeait, à droite comme à gauche, une histoire et une historiographie peu soucieuses des femmes, en particulier quand elles se mêlent de politique.

L'on rappellera par ailleurs - comme on l'a mentionné plus haut-, l'importance de l'ouvrage que l'écrivain et dramaturge Léopold Lacour consacra en 1900 aux Origines du féminisme contemporain. Trois femmes de la Révolution: Olympe de Gouges, Théroigne de Méricourt, Rose Lacombe, en se réclamant d'Alphonse Aulard, et d'une «histoire de la Révolution entrée dans sa phase scientifique " ${ }^{45}$. Cet ouvrage de vulgarisation fut décisif, peut-on penser, dans une histoire renouvelée de la prise en compte du rôle des femmes dans la Révolution, et dans la réhabilitation d'olympe de Gouges notamment, en qui l'auteur voit «le grand aïeul féminin du Féminisme intégral ${ }^{46}$ ». Mais c'est pourtant à Théroigne de Méricourt, que l'auteur consacre la part du lion en nombre de pages, faisant de l'« amazone de la Révolution » le centre de son étude. Et, forte de cette lecture, c'est aussi le personnage de "la Belle Liégeoise", et non celui d'Olympe de Gouges, que Sarah Bernhardt souhaitera incarner lorsqu'elle passe commande à Paul Hervieu d'une pièce historique, écrite pour elle et que, en décembre 1902, elle mettra personnellement en scène, dans la surenchère du grand spectacle ${ }^{47}$. Pourquoi Théroigne plutôt qu'Olympe, il y aurait beaucoup à dire, alors que ces deux figures, mal aimées des histoires de la Révolution, conjuguent à égalité, semble-t-il, le scandale d'une vie galante, des sympathies girondines, la réputation d'amazones fêlées et celle d'agitatrices intempestives... Mais leurs trajectoires n'ont pas la même signification et le cas de folie avérée de Théroigne, son internement dans les « Petites maisons » - qui lui a permis d'échapper à la guillotine, en la vouant à une mort ignoble des années plus tard - intéressait sans doute davantage les contemporains de Charcot.

À n'en pas douter, il y a bien des raisons - relevant de l'actualité historiographique et d'un contexte idéologique - qui, en 1902, faisaient de Théroigne de Méricourt un « objet théâtral » (et spectaculaire) plus pertinent. Mais, en réalité, il est une raison circonstancielle qui a fini par nous apparaître, expliquant que ce fut aussi sans doute la nouveauté d'une «Théroigne » sur les planches qui l'emporta alors. Car, en cherchant 
bien dans la production théâtrale qui précède, l'on finit tout de même par trouver une Olympe de Gouges théâtrale - et qui ne manquait pas de panache de surcroît - dans la pièce qu'Émile Moreau, jeune auteur débutant, consacra à Camille Desmoulins ${ }^{48}$, représentée à Paris en avril 1879, sur l'ancien Théâtre-Historique, alors rebaptisé Théâtre des Nations. Le futur collaborateur de Victorien Sardou pour une Madame SansGêne d'anthologie en $1893^{49}$ - et peut-être son porte-plume en d'autres occasions moins repérées ${ }^{50}$ - signait là l'un de ces drames romanesques dont Le Chevalier de Maison rouge de Dumas fournissait sans doute le modèle pour ce qui concernait l'agencement d'une intrigue de théâtre. Soucieux aussi de reconstitution historique, le jeune dramaturge proposait une succession de tableaux scéniques qui annonçait les pièces historiques à grand-spectacle dont la Belle Époque se fit une spécialité. Par cette hybridité, et du fait également des circonstances d'une réception chahutée, le drame historique de Moreau (tombé aux oubliettes de l'histoire du théâtre) préfigurait, à bien des égards, le Thermidor de Sardou de 1891.

Ce Camille Desmoulins présente l'intérêt de témoigner d'un moment de transition dans l'histoire des représentations de la Révolution au théâtre au XIXe siècle, au tournant des années 1870-1880, quand on cherchait à fonder une scène authentiquement républicaine au Théâtre des Nations, sous la direction de Gustave Bertrand. Émile Zola ne s'y trompait pas, qui scruta avec attention l'aventure éphémère de ce théâtre, dans les chroniques qu'il rédigeait à cette époque pour Le Voltaire ${ }^{51}$. Mais la pièce de Moreau, qui mélangeait les "parties fausses » et les « documents textuels ", était un « monstre " aux yeux de Zola, qui déplorait que son auteur conjuguât si maladroitement la fantaisie du romanesque et l'érudition, empêchant la réalisation d'un drame historique digne de ce nom, à même d'intéresser les citoyens de la nouvelle République en traitant véritablement de l'histoire contemporaine ${ }^{52}$.

S'inspirant de l'historiographie récente - et plus précisément des ouvrages de Jules Claretie consacrés à Camille et Lucile Desmoulins, et aux dantonistes ${ }^{53}$ - Moreau avait en effet composé les tableaux successifs d'une trajectoire politique et biographique allant de l'appel de Camille Desmoulins au Palais-Royal, le 12 juillet 1789, jusqu'à l'exécution des dantonistes Place de La Révolution le 4 avril 1794, en passant par les Cordeliers, l'arrestation nocturne de Danton et de ses amis, le procès truqué au Tribunal révolutionnaire, la Prison du Luxembourg... À des scènes et des tableaux découpés dans le récit de l'Histoire, le dramaturge avait mêlé l'artifice d'une intrigue passionnelle, au sein de laquelle Lucile se voyait doublement convoitée par le Général Dillon et par Robespierre, et où l'amour et la jalousie animant les personnages principaux, mais aussi des figures secondaires - comme la maîtresse d'Hébert ou l'hôtesse de Robespierre -, produisaient autant de rebondissements dans l'action et de perturbations dans la séquence des faits historiques. Or, parmi la galerie nombreuse des personnages de ce drame historique, exact et désinvolte à la fois, auquel l'alacrité de son dialogue en prose donnait parfois l'allure de la comédie, Moreau avait réservé une place de choix à la figure d'olympe de Gouges, dont les interventions et les bons mots relevaient à la fois de la vérité de l'Histoire et de la liberté de la fiction. Tout d'abord présentée dans la pièce en comparse un peu ridicule, puis constamment brimée par une parole masculine la faisant taire, on la voyait affirmer progressivement une toute autre stature, gagnant d'acte en acte la consistance d'une héroïne à la Dumas et l'aura d'une justicière. 
26 Au Palais-Royal, où elle était d'abord désignée par Hébert comme une «toquée qui se mêle d'écrire $~^{54}$, on la voyait passer au bras d'Hérault de Séchelles, puis s'entretenir gaiement avec le Général Dillon, avant de poursuivre un Fabre d'Églantine quelque peu fuyant, et enfin applaudir avec enthousiasme l'appel de Desmoulins, en offrant ses jarretières en guise de cocarde. Au Club des Cordeliers, on la voyait ensuite se plaindre de ses déboires théâtraux, puis monter à la tribune pour tenter en vain de faire entendre sa Déclaration des Droits de la femme et de la citoyenne ${ }^{55}$. La suite de l'intrigue, au prix d'une belle infidélité historique consistant à retarder d'un an l'arrestation et la condamnation d'olympe de Gouges - dans la contrainte mélodramatique d'un drame donné au Théâtre Historique -, faisait d'elle l'héroïque artisane d'un plan de sauvetage de Camille Desmoulins, de Danton et des leurs. C'était elle qui venait prévenir Lucile, Mme Duplessis, Mme Danton du danger guettant les dantonistes, pris entre Robespierre et les hébertistes: "Soyons hommes, citoyennes... $»^{56}$. On l'entendait à plusieurs reprises ensuite, dans l'acte du Tribunal révolutionnaire, applaudir aux propos de Danton et de Camille, et exciter en quelque sorte la foule par ses interruptions et commentaires durant l'interrogatoire public. S'étant jetée dans la gueule du loup en venant défier Robespierre, elle était ensuite incarcérée avec ses amis politiques dans la prison du Petit Luxembourg, pour leur apporter des nouvelles de l'extérieur, ainsi qu'un sauf-conduit qu'elle ne pouvait utiliser, mais qui profitait à Dillon, l'amoureux malheureux de Lucile (lequel allait tenter ensuite vainement de soustraire les dantonistes à la guillotine). Au dernier acte de la pièce, ce mouvement inattendu de réhabilitation et d'héroïsation théâtrale d'olympe de Gouges atteignait alors son sommet, dans le sacrifice qu'elle faisait de sa vie, par conviction républicaine mais aussi dans une forme de suicide et de désespoir suite à la trahison de son fils (reniement dont on sait qu'il n'eut lieu en réalité qu'après sa mort). Olympe présentée comme une héroïne se sacrifiant pour "délivrer Paris de la Terreur », la scène s'achevait sur un «Vivat Olympe ! » et encore un de ses bons mots pleins de panache : «Et les comédiens qui m'accusaient de manquer d'imagination !....1 ${ }^{57}$

Au-delà du procès théatral intenté à un jeune auteur coupable, aux yeux de la critique théâtrale, (de Zola à Vitu) de ne connaître ni les règles de l'art dramatique, ni le respect de la vérité de l'Histoire, un examen attentif de la réception critique du Camille Desmoulins de Moreau révèle aussi l'exaspération que suscita ce personnage d'olympe de Gouges, accablé par la majorité des critiques de la presse quotidienne, ou tout simplement censuré dans leurs comptes rendus. Le " Monsieur de l'Orchestre », soiriste du Figaro, s'amusait de l'apparition de l'actrice Marie Dumas au premier acte, qui tenait le rôle d'olympe dans une «robe Constitution et un chapeau à la citoyenne, surchargé de plumes multicolores, comme ceux des amazones de cirques forains $»^{58}$. Son confrère, le très conservateur Auguste Vitu, disqualifiait brutalement, et dans le même mouvement misogyne, le personnage d'olympe de Gouges et l'actrice Marie Dumas qui s'était fait connaître pour son action en faveur des répertoires étrangers et était alors une sorte d'éminence grise au Théâtre des Nations ${ }^{59}$ :

À travers le drame décousu de M. Émile Moreau, circule, sans l'ombre d'utilité, de raison, ni d'amusement le personnage d'olympe de Gouges, qui fut, parmi les femmes de la Révolution, ce qu'a été M. Gagne pour la génération présente. Madame Marie Dumas, chargée de ce rôle, n'y a point réussi... Le public a paru convaincu que Madame Marie Dumas, conférencière à la parole abondante et actrice supportable dans des matinées consacrées plutôt à la curiosité littéraire qu’à la bonne exécution théâtrale, devra se résigner à n'aborder la scène qu'en «amateur » pour son agrément personnel ${ }^{60}$. 
même condescendance anti bas-bleus s'exprimait chez Francisque Sarcey, qui ironisait lui aussi sur le personnage d'olympe et sur l'activité de conférencière de l'actrice :

On avait taillé dans le drame, exprès pour Mlle Marie Dumas, un rôle qui est encore plus inutile qu'il n'est long. Si nous avions un conseil à donner à cette spirituelle artiste, qui ne sera jamais comédienne, ce serait de profiter de son remarquable talent de paroles pour abandonner le théâtre et devenir, en se faisant conférencière, notre collègue dans cet art difficile. Tout le monde y gagnerait ${ }^{61}$.

Thomas Grimm, dans Le Petit Parisien - moins grincheux, mais reprochant à l'auteur d'avoir mis trop d'intrigue dans sa mise en scène de la lutte des dantonistes contre les robespierristes et les hébertistes - se plaignait également de la longueur du rôle :

Mlle Marie Dumas tient le rôle épisodique d'olympe de Gouges, la muse tâtonnante de la Révolution, avec désinvolture; ce rôle est seulement beaucoup trop développé ${ }^{62}$.

La misogynie de François Oswald, dans Le Gaulois, était plus explicite :

En somme la représentation d'hier a été une désillusion. On s'attendait à quelque chose de crâne, de hardi ; on s'est trouvé en présence de héros larmoyants qui n'ont pas le courage de leur opinion et de leurs actes. C'est un drame médiocre, qui n'a d'éclatant que le titre et d'intéressant que l'interprétation. Mlle Léonide Leblanc est touchante sous les traits de Lucile, et Mlle Marie Dumas joue avec conscience un rôle insupportable, celui d'Olympe de Gouges ${ }^{63}$.

31 Enfin Alphonse Daudet - encore lui - trouvait une nouvelle occasion de broder sur le « tempérament » de la méridionale :

Mme Marie Dumas n'a su nous rendre que bien imparfaitement cette étrange Olympe de Gouges, dont nous avons - il n'y a pas bien longtemps - esquissé un vague croquis pour nos lecteurs, la prolixe montalbanaise, parfumée d'ail et de bergamote, mélange d'afféterie et de grossièreté, crâne fêlé, cervelle en ébullition comme le Midi seul peut en cuire dans sa cuve toujours fumante ${ }^{64}$.

Ce florilège plaisamment caricatural, témoignage de la persistance du préjugé antiOlympe au XIX ${ }^{\mathrm{e}}$ siècle, plaide a contrario en faveur de la belle pièce de jeunesse d'Émile Moreau, où le dramaturge confiait à la fiction théâtrale le sort d'une figure décriée en réinventant son personnage pour mieux le faire entendre. Sans craindre les libertés prises avec l'histoire, il thématisait avec humour l'histoire d'une longue censure. Dans le Camille Desmoulins de Moreau, un drame en cachait un autre, et une Olympe de Gouges se jouait en creux de l'action principale, crypto-pièce ou œuvre seconde dans laquelle le dramaturge, exhibant nombre de préjugés dont l'olympe historique était la victime persistante, dénonçait audacieusement - et en actes ! - sa mauvaise réputation, en s'efforçant de rendre sa vérité au personnage grâce aux pouvoirs paradoxaux d'une fiction de théâtre. En choisissant d'enrôler Olympe dans le camp des dantonistes, en confiant son rôle à la femme de tête qu'était Marie Dumas, en lui taillant un rôle d'aventurière à panache, il contribuait avant l'heure à forger le mythe d'une Olympe de Gouges en attente du Panthéon d'une république féminisée.

C'est cette dimension - inaperçue, ou trop bien comprise par les contemporains - qui constitue à nos yeux, et de manière inattendue, l'originalité d'une pièce et d'un spectacle fin-de-siècle - généralement décriés dans la presse de l'époque, à droite comme à gauche. Parce qu'ils dérogeaient trop évidemment à ce que les uns et les autres attendaient d'un drame historique sur la Révolution, cette Olympe de Gouges 
insolemment romanesque et revendicative n'apparaissait pas comme la moindre des fautes de goût commises par son auteur et passager intercesseur.

\section{Conclusion}

Personnage à éclipses s'il en fut, celle que l'on a désigné comme «la grande révolutionnaire inconnue $»^{65}$ a troqué aujourd'hui sa mauvaise réputation contre un statut de pionnière du féminisme. Pour méritée que soit cette requalification du personnage et de son action, elle tend parfois à gommer les aspérités d'un parcours singulier au fil duquel la figure d'olympe de Gouges parait défier les tentatives de saisie trop normalisante, et celles d'assignations (politiques notamment) trop précises. Entre les avancées de la "vérité » historique (qui resituent Olympe de Gouges dans son temps) et les utiles enrôlements contemporains, il y a place pour une histoire des représentations imaginaires d'une femme qui a eu recours elle-même aux moyens de la littérature et du théatre pour se mettre en scène et pour inventer la langue et les formes d'une action singulière, et ainsi traverser le temps envers et contre tout. Ce tropisme théâtral d'olympe de Gouges qui nous a intéressée ici est certainement l'une des clefs qui donnent accès au personnage - sa légende et sa vérité tout à la fois - et c'est peut-être la leçon rétrospective intéressante qu'un drame historique oublié de la fin du XIX $x^{e}$ siècle nous donne à comprendre et à goûter.

\section{NOTES}

2. Voir notamment Olympe DE GOUGES, L'Homme généreux, drame en cinq actes et en prose, 1786 ; ou bien ID., La France sauvée, ou le Tyran détrôné, drame en cinq actes en prose, 1792.

3. Léopold Lacour décrit bien cette activité d'«ardente publiciste» dans la première monographie sérieusement documentée consacrée à Olympe de Gouges en 1900 : Léopold LAcour, Trois femmes de la Révolution: Olympe de Gouges, Théroigne de Méricourt, Rose Lacombe, Paris, PlonNourrit, 1900, p. 55. Olivier Blanc (auteur de nombreux ouvrages sur Olympe de Gouges, depuis une biographie pionnière publié chez Syros en 1981) y voit un "modèle inédit d'engagement citoyen » : Olivier BLANC, « Olympe de Gouges, un nouveau modèle d'engagement citoyen »), dans Martial Poirson (dir.), Amazones de la Révolution, Des femmes dans la tourmente de 1789, Montreuil, Gourcuff Gradenigo, 2016, p. 69 (voir aussi Olympe DE GOUGES, Écrits politiques, préfacés par Olivier Blanc, 2 volumes, Indigo \& Côté Femmes éditions, 2014).

4. L'historienne et anthropologue Nicole Pellegrin, analysant la parole d'Olympe de Gouges, souligne ce fait et le relie de manière éclairante à sa pensée politique, et notamment à son "fédéralisme» dans sa communication du colloque "Olympe de Gouges, une femme du $\mathrm{xxI}^{\mathrm{e}}$ siècle", colloque soutenu par Le Monde diplomatique, l'Unesco et la Ville de Montreuil, 14-15 novembre 2008. L'enregistrement de son intervention est accessible sur la page «Écouter le colloque Olympe de Gouges » $\mathrm{du}$ site $\mathrm{du}$ Monde diplomatique: https://www.mondediplomatique.fr/document/olympedegouges

5. Voir notamment Olympe DEGOUGES, «Préface sans caractère », op.cit., p. 136: « Mais je suis l'élève de la nature ; je l'ai dit, je le répète, je ne dois rien aux connaissances des hommes : je suis 
mon ouvrage, et lorsque je compose, il n'y a sur la table que de l'encre, du papier et des plumes. Très souvent, j'ai de mauvais secrétaires qui multiplient les fautes au lieu de les corriger ». Voir aussi sa préface du Mariage inattendu de Chérubin: "La passion qui me domine pour créer de nouveaux sujets, me fait oublier ceux qui les ont précédés; l'activité de dix secrétaires ne suffirait pas à la fécondité de mon imagination. J'ai trente pièces au moins ; je conviens qu'il y en a beaucoup plus de mauvaises que de bonnes; mais je dois convenir aussi que j'en ai dix qui ne sont pas dépourvues du sens commun", dans EFuvres de Madame de Gouges (dédiées à Monseigneur le Duc d'Orléans), tome premier [Mémoires de Mme de Valmont; L'homme généreux, drame; Le Mariage inattendu de Chérubin, comédie], Chez L'Auteur et Cailleau imprimeur libraire, 1788.

6. Léopold LACOUR, op. cit., p. 42 : « Pièces de théâtre, romans, brochures politiques, placards, tout fut dicté. On a un petit billet de sa main aux Archives Nationales; mais l'écriture de ce billet, les signatures qui se trouvent au même carton, et qui, d'ailleurs, varient - tantôt elle signe de Gouge ou de Gouges avec Olimpe ou Olympe, tantôt Olimpe Degouges - témoignent assez que le maniement de la plume lui fut jusqu'à la fin très pénible. Il s'est vendu à différentes époques des lettres d'elle ; nous ne les connaissons pas, mais l'Amateur d'autographes nous en est garant: une seule excepté [sic], d'une page, entièrement de la main d'olympe, il n'y avait d'autographe dans les autres que la signature. - Même arrêtée, de la Mairie où elle est détenue, elle dicte ses lettres ; elle les dictera dans ses deux prisons, enfin à la Conciergerie, le 2 novembre après sa condamnation, et le 3 où elle fut décapitée. Sa dernière lettre, - à son fils, pour lui crier parmi ses larmes, son désespoir de mère,- elle essaiera il est vrai de la finir elle-même Elle tracera difficilement quelques lignes, une dizaine, d'une orthographe encore pire que l'écriture. »

Le constat de Lacour, sans doute trop catégorique, a le mérite d'attirer l'attention sur la dimension performative de la création littéraire d'Olympe de Gouges, confirmée par Olivier BLANC (dans Olympe de Gouges, op. cit. p. 44).

7. Le présent article est la version écrite et développée d'une communication consacrée à "Olympe de Gouges et la scène, trajectoire d'une incarnation ", prononcée lors d'une intervention collective, auprès de mes collègues Florence Lotterie et Olivier Ritz, dans le cadre du colloque international «La Révolution en 3D (textes, images, sons). 1787-2440 », Anne Simonin et Pierre Serna (dir.), université Paris 1 Panthéon-Sorbonne, 14-16 mars 2019. C'est en qualité de responsables du séminaire IMAREV 18-21 - Imaginaires de la Révolution française de 1789 à nos jours (université de Paris, CERILAC, Centre Seebacher) que nous sommes intervenus dans une communication en triptyque, intitulée "L'objet "Olympe de Gouges" », faisant le choix d'une illustration monographique en trois temps, inscrite dans les logiques disciplinaires qui sont celles d'IMAREV : https://imarev.hypotheses.org : voir dans ce même numéro Florence LOTTERIE, « Une fiction de la Révolution : la projet de "la Tenture Olympe de Gouges" (2009- ?)»; et Olivier RITZ «Le sacre retardée d'une écrivaine : Olympe de Gouges ».

8. Comme dans le titre du roman de Paul Scarron, nous employons délibérément ce terme ici, dans son sens littéraire et ancien référant à tout ce qui a trait à la vie du théâtre (une des passions avérées d'Olympe de Gouges, avec la politique). Mais nous assumons aussi l'acception générique plus circonscrite du terme, comme le sens élargi et contemporain de l'adjectif - ce qui ressort de la comédie, et qui fait rire-, susceptible de désigner alors secondairement et implicitement la charge des préjugés qui ont durablement accompagné la légende théâtrale d'Olympe de Gouges, au XIX siècle (et au-delà), comme nous le suggérons dans la première partie de cette étude.

9. Voir notamment Olivier BLANC, Marie-Olympe de Gouges, une humaniste à la fin du XVIII siècle, éditions René Viénet, Belaye, 2003. Voir aussi Olympe DE GOUGES, Théâtre politique, préfaces de Gisela Thiele-Knobloch, 2 volumes (I- Le Couvent, ou les vœux forcés, Mirabeau aux Champs-Elysées, L'Entrée de Dumouriez à Bruxelles, ou Les Vivandiers; II- L'Homme généreux, Les démocrates et les 
aristocrates, ou Les Curieux du Champs de Mars, La Nécessité du divorce, La France sauvée ou le Tyran détrôné), Paris, Indigo \& Côté Femmes éditions, 2014-2015.

À compléter par cet autre volume indispensable : Olympe DE GOUGES, Cuuvres complètes, t. 1 Théâtre [douze pièces, drames et comédies en prose, écrites entre 1783 et 1793, dont deux inédites], édition et introduction de Félix-Marcel Castan, Montauban, Cocagne, 2013. À quoi il convient de rajouter: Olympe DEGOUGES, L'Esclavage des nègres (version inédite du 28 décembre 1789), présentation et étude de Sylvie Chalaye et Jacqueline Razgonnikoff, coll. " Autrement même ", Paris, L'Harmattan, 2006 ; et ID., L'Heureux naufrage, ou l'esclavage des noirs (version de 1792), préface d'Eleni Varikas, Paris, Indigo \& Côté Femmes éditions, 2014.

10. Cuvres de Madame de Gouges (dédiées à Monseigneur le Duc d'Orléans), op. cit.

11. Voir Olympe DE GOUGES, Le Couvent ou les vœux forcés, drame en trois actes, à Paris, à Paris, Chez La veuve Duchesne, La Veuve Bailly, et Chez les marchands de nouveautés, mars 1792.

12. Charles-Guillaume ÉTIENNE et Alphonse MARTAINVILLE Histoire du Théâtre Français, depuis le commencement de la Révolution jusqu'à la réunion générale, Paris, Barba, 1802, tome premier, p. 58-59: « Malgré l'enthousiasme national, la comédie, ou plutôt le drame, intitulé : L'Esclavage des Nègres, représenté le 27 décembre, n'obtint aucun succès. [...] L'auteur de cet ouvrage est Madame De Gouge. Sans égard pour le beau sexe, le public le siffla impitoyablement ». Ibid., tome 3, p. 64-68: «Rien de plus bizarre, de plus extravagant que cet ouvrage, dont tout le mérite consistait dans des marches, combats et évolutions militaires ».

13. Ibid., tome 3, p. 65.

14. Voir les Mémoires de Fleury de la Comédie Française (1757-1820), Ambroise Dupont éditeur, 1835-1836, tome IV, ch. 5, p. 233-275.

15. Ibid., p. 237.

16. Charles-Guillaume ÉTIENNE et Alphonse MARTAINVILLE Histoire du Théâtre Français..., op. cit.

17. Marvin CARLSON, Le théâtre de la Révolution française, de Marvin Carlson (1966, traduit en 1970 par J. et L. Brant), Gallimard, p. 74-75: «Olympe de Gouges, vieille dame originale douée de l'étrange talent d'écrire sur des sujets anodins, des pièces qui toutes faisaient fiasco, composa un Henri IV et le jeune Desilles qui n'eut aucun succès ».

18. Eugène JAUfFRET, Le Théâtre Révolutionnaire (1788-1799), Paris, Furne, 1869, p. 219 : « À quelques jours de là ( 23 janvier), le théâtre de la rue Richelieu eut aussi sa petite émeute, mais plus burlesque que tragique: une mauvaise comédie en prose, intitulée Le Général Dumouriez à Bruxelles, en fut l'occasion. L'auteur était une femme, Olympe Degouges, qui s'était offerte, lors du procès de Louis XVI, pour défendre ce prince. C'était un ouvrage extravagant, chargé d'incidents ridicules, rempli de portraits grotesques et de fanfaronnades. [...] Au moment où Mlle Candeille s'avançait pour nommer l'auteur, une femme vieille et laide s'écria d'une première loge: «Citoyens, vous demandez l'auteur; le voici. C'est moi, Olympe Degouges. Si la pièce vous a paru mauvaise, c'est que les acteurs l'ont très mal jouée ». Régulièrement " recopié » au XIXe siècle, le récit de cette "représentation tumultueuse » est cité, par exemple, dans le "Courrier des théâtres » du journal Le Figaro, jeudi 22 février 1872. On le retrouve intégralement dans Marvin CARLSON, op. cit., pp. 177-178: «Le 23 janvier, le Richelieu offrit son tribut aux sentiments belliqueux du jour dans L'Entrée de Dumouriez à Bruxelles. L'auteur était Olympe de Gouges, vieux laideron irascible dont les échecs avaient montré que les pièces de circonstances n'étaient pas nécessairement assurées du succès, fût-ce dans le climat favorable du Paris de la Révolution [...] L'Entrée de Dumouriez à Bruxelles comportait surtout des marches, des combats, et des manœuvres militaires. L'étrangeté et la confusion de l'intrigue provoquèrent l'hilarité du début à la fin [etc.]».

19. Jean-Baptiste CAPEFIGUE, Les Déesses de la liberté. Les femmes de la Convention et du Directoire, Paris, Amyot, 1862. 
20. Charles MONSELET, Les Oubliés et les Dédaignés du Dix-huitième siècle, Paris, Charpentier, 1876, chap. IV « Olympe de Gouges », p. 99-123

21. Ibid., p. 99.

22. Ibid. p. 103-105

23. Ibid., p. 114-116.

24. Alphonse DAUDET, L'Événement ou Le Figaro, mai 1876, cité dans Id., Chroniques dramatiques, édition A. S. Dufief, Champion, 2006, p. 522-526.

25. Ibid.

26. Dans Le Monde des théâtres pendant la Révolution 1789-1800 (Perrin, 1922), Jacques HÉRISSAY, s'inspirant lui aussi de près des Mémoires de Fleury, consacre quelques pages intéressantes à cette «femme étrange, détraquée illustre, connue dans l'histoire sous le pseudonyme d'olympe de Gouges » («Les auteurs dramatiques et la Terreur », p. 192-196).

27. Pour compléter cet échantillon d'une historiographie minimale et durablement misogyne réservée au personnage d'Olympe de Gouges, l'on peut enfin se référer à la notice que lui consacre Gérard Walter, en 1952, dans son édition de L'Histoire de la Révolution Française de Michelet, et à ce propos expéditif : "Michelet, faisant preuve d'une délicatesse infinie, a su rendre sympathique et presque touchante cette quadragénaire hystérique, à qui son veuvage semble avoir pesé un peu trop lourd», dans Michelet, Histoire de la Révolution Française, Bibliothèque de La Pléiade, Gallimard, 1952, T. 2, p. 1247.

28. Léopold LACOUR, "Olympe de Gouges ", dans Les origines du féminisme contemporain. Trois femmes de la Révolution: Olympe de Gouges, Théroigne de Méricourt, Rose Lacombe, Paris, Plon, 1900. p. 1-92. Dans cette étude importante, et charnière dans l'histoire de la réhabilitation d'olympe de Gouges, le critique socialiste et féministe retrace en près de quatre-vingt pages le parcours d'Olympe de Gouges en rendant justice à ses idées novatrices, sur le plan social et politique, et tente une vraie analyse de l'évolution de ses positions politiques pendant la Révolution. Mais il ne peut s'empêcher de la présenter comme une "originale qui fut tantôt folle, tantôt voyante", «une agitée et non une femme d'action ». Et son récit biographique tendancieux - mais très documenté - ne fait pas une présentation très flatteuse de son œuvre littéraire et théâtrale.

https://gallica.bnf.fr/ark:/12148/bpt6k8630585q.texteImage

29. Voir aussi, dans le présent dossier, Olivier RITZ, « Le sacre retardé... », art. cité.

30. Voir Olivier BLANC, Marie-Olympe de Gouges, une humaniste de la fin du XVIII ${ }^{\mathrm{e}}$ siècle, René Viénet, 2003 ; et ID., Olympe de Gouges, des droits de la femme à la guillotine, Tallandier, 2014.

31. Voir notamment Olympe DE GOUGES, Écrits politiques, t. 1 (1789-1791) et t. 2 (1792-1793), op. cit. Voir aussi ID., Théâtre politique, t. 1 et 2, op. cit.; et ID., CEuvres complètes, T. 1 Théâtre, op. cit. À quoi il convient de rajouter: ID., L'Esclavage des nègres, op. cit. ; et L'Heureux naufrage... (version de 1792), op. cit.

32. La pièce a notamment fait l'objet d'une lecture au Studio-théâtre de la Comédie-Française, le 18 octobre 2004 (Cycle Comédie-Française : Les Temps retrouvés, J. Huthwohl dir. ). Voir, à ce sujet et plus généralement, l'excellente introduction à la pièce d'Olympe de Gouges qu'ont donnée Sylvie CHALAYE et Jacqueline RAZGONNIKOFF, à leur édition critique de L'Esclavage des nègres, op. cit., p. VII-XXVI.

33. Voir Olympe de Gouges aux enfers, écrits sur le théâtre, brochure éditée en janvier 2012, à Toulouse (éditions La Brochure, 2012) : cette petite anthologie, réalisée à l'initiative du musée Olympe de Gouges de Montauban et sans nom d'auteur (sans doute la conservatrice, ou bibliothécaire, ou quel que soit son titre, la préposée au Fonds « Olympe de Gouges » déposé à la Bibliothèque municipale de Montauban), est sans doute la meilleure idée de réalisation théâtrale consacrée à la figure et à l'œuvre d'olympe de Gouges. Voilà ce que l'on lit sur le quatrième de couverture: "Olympe de Gouges a tenté d'être auteure de théâtre. Elle a rencontré mille difficultés. Elle a imaginé alors écrire une pièce dont elle serait le cœur et qu'elle appellerait 
Olympe aux enfers. En reprenant l'essentiel de ses préfaces, c'est exactement la sensation que l'on a : Olympe était une indésirable sans pourtant être une extrémiste. Voici ses propos à la première personne.» L'idée de cette anthologie s'inspire des écrits mêmes d'olympe de Gouges, et notamment de sa préface à Mirabeau aux Champs Élysées, comédie en un acte en prose (jouée le 15 avril 1791) : «Les propos injurieux qu'on a répandus sur mon compte ; la noire calomnie que l'on a employée pour empoisonner tout ce que j'ai fait de méritoire, seraient propres à me donner de l'orgueil, puisqu'il est vrai qu'on me traite et qu'on me persécute en grand-homme; si je pouvais me le persuader, je réaliserais le projet que j'ai formé de me retirer entièrement de la société, d'aller vivre dans la solitude, étudier nos auteurs, méditer un plan que j'ai conçu en faveur de mon sexe, de mon sexe ingrat; je connais ses défauts, ses ridicules, mais je sens qu'il peut s'élever un jour ; c'est à cela que je veux m'attacher. Cet ouvrage est de longue haleine, et je ne le présenterai pas du matin au soir; je veux faire cependant les adieux comiquement à mes concitoyens ; après avoir mis les morts au théâtre, je veux y mettre les vivants ; je veux me jouer moi-même, ne point faire grâce à mes ridicules pour ne point épargner ceux des autres, je n'ai pas trouvé de plus vaste plan, ni de plus original que Mme de Gouges aux enfers » (p. 113) .

34. On peut mentionner encore et plus récemment: Olympe de Gouges - Robespierre, une histoire de la Révolution Française, de Sandrine GAUviN, mise en scène par Sandrine Gauvin, avec Isabelle Couloigner, Adrien Deschamps, Mélisa Lucchini, Manon Simier. Spectacle créé en mars 2019 dans le cadre du mois des droits des femmes à l'Espace Culturel Dispan De Floran à L'Haÿ-les-Roses, et qui aurait dû se jouer de décembre 2020 à mars 2021 au Folie-Théâtre à Paris : «1789, la colère gronde! Le peuple se soulève! La Révolution Française est en marche et voit s'affronter Olympe de Gouges, femme de lettre et féministe, et Robespierre, avocat et homme politique. Animés par les mêmes combats, ils s'opposent sur la tournure des événements. La confrontation commence avec d'un côté Olympe de Gouges et Sophie de Condorcet et de l'autre Robespierre et Eléonore Duplay. Pendant ce temps, Marie-Antoinette tente de fuir le pays. "

35. Elsa Solal, Olympe de Gouges : « Non à la discrimination des femmes », Arles, Actes Sud, 2014, $96 \mathrm{p}$.

36. Elsa SolAL, Olympe de Gouges, Manage, Lansman éditeur, Coll. " Théâtre à vif », 2018, 35 p.

37. Il existe une captation vidéo du spectacle (cote BnF : NUMAV 1148698); ou chez L'Harmattan, en DVD ou VOD ( https://www.editions-harmattan.fr/index.asp? navig $=$ catalogue $\& o b j=v i d e o \& n o=2729$ ).

38. Annie VERGNE et Clarissa PALMER, Olympe de Gouges porteuse d'espoir, d'après les écrits d'Olympe de Gouges ; Olympe de Gouges, a beacon of hope, based on the writings of Olympes de Gouges, L'Harmattan, 2012.

39. Voir https://www.theatre-contemporain.net/spectacles/Olympe-de-Gouges-porteuse-despoir.

40. Le spectacle était programmé au Théâtre de L'Épée de bois, à la Cartoucherie de Vincennes, du 27 février au 8 mars 2020. La tournée prévue - après la longue paralysie de la vie théâtrale due à la crise sanitaire de la Covid 19 - reprend de juin à décembre 2021, dans plusieurs villes de France.

41. Les quatre comédien'n·e's à la création étaient Catherine Anne (la prisonnière), Luce Mouchel (la mère), Morgane Real (la jeune femme), et Pol Tronco (le jeune soldat).

42. Voir sur le site de la Compagnie de Catherine Anne, "À Brûle-pourpoint ", le dossier complet du spectacle, des extraits vidéo, l'entretien audio réalisé avec l'artiste, et une revue de presse. Voir aussi les extraits du spectacle sur https://www.theatre-contemporain.net/spectacles/j-aireve-la-revolution.

43. Dans sa postface de la pièce, publiée en 2018 aux édition Actes-Sud papier, Catherine Anne dédie son texte à Hadel Hakim, disparu en 2017, metteur en scène et ancien co-directeur du Théâtre des quartiers d'Ivry. 
44. À titre d'exemples: Madame Roland, drame historique en trois actes, mêlé de chant, de Madame Ancelot, représenté le 28 octobre 1843 au Théâtre du Vaudeville [Paris, Beck, 1843]; Charlotte Corday, tragédie en cinq actes en vers, de François Ponsard, représentée le 23 mars 1850 au Théâtre-Français (reprise en 1880, 1889 etc.), [Paris, Blanchard, 1850] ; Manon Roland, drame en vers en cinq actes (en société avec M. Camille de Sainte-Croix), représenté à la Comédie Française, le 4 mai 1896 [Théâtre d'Emile Bergerat, vol.4, Paris, Ollenforff, 1900]; Lucile Desmoulins, drame en vers, en cinq actes et huit tableaux, de Jules Barbier, représenté au Théâtre de la République le 6 novembre 1896 [Lévy, 1896]; Madame Tallien, pièce historique en cinq actes et huit tableaux de MM. Paul Berthelot et Claude Roland, musique de Jane Vieu, représenté à

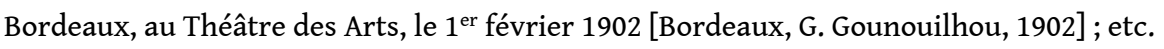

45. Léopold LACOUR, Les origines du féminisme contemporain..., op. cit. (voir l'« Avant-propos », p. V).

46. Ibid., p. 5.

47. Paul HERVIEU, Théroigne de Méricourt, pièce en six actes en prose, représentée au Théâtre Sarah Bernhardt le 23 décembre 1902, Paris, Lemerre, 1902, 264 p. Voir Sophie LUCET, « Les Fantômes de la Révolution, ou les leurres du grand spectacle », dans Florence Baillet, Mireille Losco, Arnaud Rykner (dir.), L'œil au théâtre, Presses universitaires de Louvain, 2017, p. 94-105.

48. Émile MOREAU, Camille Desmoulins, drame en cinq actes et huit tableaux, Paris, Tresse, 1879 (Théâtre-Historique, le $1^{\mathrm{er}}$ avril 1879, musique de Victor Chéry, décors de Nézel et Poisson), trente-cinq représentations, dont une en matinée le 14 avril.

49. Victorien SARDOU et Émile MOREAU, Madame Sans-Gêne, comédie historique précédée d'un prologue, L'Illustration théâtrale, 21 décembre 1907. Voir l'édition scientifique du texte par Marion CHÉNETIER dans Isabelle Moindrot (dir.), Drames et pièces historiques de Victorien Sardou, vol. 4, Paris, Classiques Garnier, 2017.

50. Émile Moreau a collaboré à plusieurs reprises avec Victorien Sardou : Cléopâtre, drame en cinq actes et six tableaux, représenté au Théâtre de la Porte-Saint-Martin en 1890 ; Madame-Sans Gêne, comédie historique en trois actes et un prologue représenté au Théâtre du Vaudeville en 1893 ; Dante, drame en cinq actes, et 12 tableaux représenté au Théâtre de Drury Lane (à Londres) en 1903, dans une traduction de Laurence Irving - pour ces trois pièces, voir Émile MOREAU, Théâtre, vol.1, Ollendorff, 1913 -; enfin Madame Tallien, drame en cinq actes et six tableaux, œuvre posthume de Victorien Sardou, achevée par Émile Moreau, et représentée au Théâtre Lyrique de Milan en 1912, dans une traduction de Targioni-Tozetti. Moreau signe aussi, en 1891, un court récit «adapté » du Thermidor de Victorien Sardou pour accompagner un photo-programme du spectacle («Le Théâtre instantané », Dentu, 1891), ainsi qu'un « Compte-rendu analytique » de la pièce (Nilson, 1892), puisque son auteur refuse à cette époque, et pour des questions de droits, de publier les textes de ses pièces. À propos de Thermidor, voir l'édition scientifique de la pièce par Sophie LUCET dans Isabelle Moindrot (dir.), Drames et pièces historiques de Victorien Sardou, op. cit., vol. 3.

51. Les chroniques théâtrales écrites par Zola dans Le Voltaire en 1878-1880 (et dans Le Bien public en 1876-1878) furent rassemblées ensuite dans Émile zolA, Le Naturalisme au théâtre, Les théories et les exemples, Paris, Charpentier, 1881.

52. Voir Sophie LUCET, « La Révolution à la scène : l'introuvable drame national du théâtre fin-desiècle ", dans Corinne Saminadayar-Perrin et Jean-Marie Roulin (dir.), Fictions de la Révolution, Saint-Étienne, Presses universitaires de Saint-Étienne, 2017, p. 255-272.

53. Camille DESMOULINS, CEuvres, recueillies et publiées d'après les textes originaux, augmentées de fragments inédits, de notes et d'un index, et précédées d'une étude biographique et littéraire, par Jules Claretie, Paris, Charpentier, 1874 ; Jules CLARETIE, Camille Desmoulins, Lucile Desmoulins : étude sur les Dantonistes : d'après des documents nouveaux et inédits, Paris, Plon, 1875.

54. Emile MOREAU, Camille Desmoulins, op. cit., Acte I, scène 2, p. 3.

55. Ibid., Acte II, scène 7, p. 21. 
56. Ibid., Acte III, scène 2, p. 25.

57. Ibid., Acte V, scène 4, p. 45.

58. « Un Monsieur de l'orchestre » [alias Arnold Mortier], Le Figaro, 2 avril 1879.

59. Marie Dumas avait lancé ses « Matinées caractéristiques » au Théâtre de la Gaité ; c'est à son instigation que Gustave Bertrand avait pris la succession de Castellano au Théâtre Historique, rebaptisé par elle Théâtre des Nations, où elle espérait poursuivre son action pédagogique, tout en contribuant à faire jouer un nouveau répertoire ; malheureusement le projet tourna court faute de succès, d'argent, et à cause de la mort prématurée de Gustave Bertrand. Voir, à ce sujet, Albert MOUSSET, «Ce que fut le Théâtre des Nations il y a trois quart de siècle », Le Monde, 23 mai 1958.

60. Auguste VITU, Le Figaro, 9 avril 1879. Le « M. Gagne» auquel le critique se réfère est Paulin Gagne, républicain mystique et fou littéraire, auteur notamment de L'Unitéide ou la femme messie, poème universel en douze chants, publié en 1857.

61. Francisque SARCEY, Le Temps, 7 avril 1879.

62. Thomas GRIMM, Le Petit Parisien, 4 avril 1879.

63. François OSWALD, Le Gaulois, 3 avril 1879.

64. Alphonse DAUDET, Journal officiel, 7 avril 1879.

65. La formule est de Gilles Manceron, citée par Benoîte Groult dans une communication prononcée en 2008, durant le colloque «Olympe de Gouges, une femme du XxI siècle », colloque soutenu par Le Monde diplomatique, L'Unesco, La Ville de Montreuil, 14-15 novembre 2008, https:// www.monde-diplomatique.fr/document/olympedegouges

\section{RÉSUMÉS}

Portant sur les représentations imaginaires d'olympe de Gouges et sur sa postérité théâtrale, cette étude traite tout d'abord de la mauvaise réputation du personnage, entretenue tout au long $\mathrm{du} \mathrm{XIX}^{\mathrm{e}}$ siècle, aux dépens de son œuvre littéraire et de son action politique. L'étude de trois spectacles du début $\mathrm{du} \mathrm{xxI}^{\mathrm{e}}$ siècle permet ensuite d'aborder le «devenir théâtre » d'olympe de Gouges, dans les formes parfois convenues de l'héroïsation et de la célébration d'une pionnière de l'histoire des femmes. La troisième partie analyse un spectacle oublié du XIX siècle : le Camille Desmoulins d'Émile Moreau (futur collaborateur de Victorien Sardou), créé en 1879 au ThéâtreHistorique. Dans ce drame, l'auteur fait surgir une Olympe de Gouges remarquable et prophétique, d'une efficacité théâtrale que ne lui pardonnent pas les critiques contemporains, inquiets du retour de cette figure refoulée par l'Histoire et qui vient avant l'heure réclamer ses droits dans une fiction de théâtre.

Focusing on the imaginary representations of Olympe de Gouges and on her theatrical posterity, this study deals first of all with the ill repute of the character, maintained throughout the 19th century, at the expense of her literary work and her political action. Afterwards, the study of three plays from the beginning of the 21stcentury will allow us to approach the metamorphosis of Olympe de Gouges into a theatrical figure, in the sometimes conventional forms of heroisation and celebration of a pioneer of women's history. The third part analyses a forgotten play of the 19th century: Camille Desmoulins, by Émile Moreau (future collaborator of Victorien Sardou), created in 1879 at the Théâtre-Historique. In this drama, the author brings 
into existence a remarkable and prophetic Olympe de Gouges, with a theatrical efficiency that contemporary critics could not accept, troubled by the return of this figure repressed by History and who, before her time, lays claim to her rights in a theatrical fiction.

INDEX

Keywords : French Revolution, Olympe de Gouges, Theater, Drama, Feminism, Gender Mots-clés : Révolution Française, Olympe de Gouges, Théâtre, Drame, Féminisme, Genre

\section{AUTEUR}

\section{SOPHIE LUCET}

CERILAC - EA 4410

Université de Paris 